\title{
Study of the relationship between AGEs and oxidative stress damage to trophoblast cell mitochondria
}

\author{
Lingling Jiang, Jianying Yan, lixiang Wu \\ Fujian Provincial Maternity and Children Hospital, Fuzhou, Fujian, China
}

\begin{abstract}
Objectives: To study the influence of AGEs on placental trophoblast mitochondria oxidative stress, and to explore the possible pathogenesis which may participate in pre-eclampsia.

Material and methods: Human trophoblast cells from early pregnancy were cultured by an enzyme-digestion method. When trophoblast cells reached approximately $70-80 \%$ after passages, they were incubated with pre-eclampsia serum for 24 hours. A fluorescent dye assay was applied to measure the mitochondrial membrane potential; ELISA was used to measure the activity of the mitochondrial permeability transition pore. mtDNA was detected by Real-time fluorescence quantitative Reverse Transcription-Polymerase Chain Reaction (RT-PCR). We continued to culture one group of cells with pre-eclampsia maternal serum, and other cells were pulsed with $600 \mathrm{mg} / \mathrm{L}$ AGEs. Cells were incubated for 16 hours before assaying the levels of mitochondrial oxidative stress damage.

Results: The levels of mitochondria oxidative stress damage in the AGEs group were higher than in the pre-eclampsia group 1 and pre-eclampsia group 2 . There was no statistically significant difference in mitochondrial oxidative stress damage between the pre-eclampsia group 1 and group 2 .

Conclusions: The AGEs are involved in the pathogenesis of pre-eclampsia, possibly through the enhancement of mitochondrial oxidative stress damage.
\end{abstract}

Key words: pre-eclampsia, advanced glycation end products, oxidative stress, trophoblast cell

Ginekologia Polska 2017; 88, 7: 372-378

\section{INTRODUCTION}

Pre-eclampsia is a hypertensive disorder which occurs only during pregnancy and is a major cause of maternal-fetal morbidity and mortality. The mechanisms responsible for pre-eclampsia are not completely understood. It is generally accepted that oxidative stress may be one of the important mechanisms in the pathophysiology of pre-eclampsia.

Advanced glycation end products (AGEs) are formed when a reducing sugar, such as glucose, reacts non-enzymatically with free amino groups in proteins, lipids and nucleic acids, spontaneously reacting with the aldehyde groups or ketone groups of glucose or other reducing sugars, then forming stable end products. Chekir et al. [1] have found that the expression of AGEs in maternal serum and placenta of patients with pre-eclampsia was significantly higher when compared to the expression in a normal pregnancy. However, the exact mechanisms responsible for pre-eclampsia are not completely understood. Several previous reports [2-3] have found that accumulation of AGEs can induce reactive oxygen species (ROS) generation, mitochondrial oxidative stress damage, damage to the integrity of the mitochondria, opening the mitochondria permeability transition pore (MPTP), and decreased mitochondrial membrane potential. Zusterzeel et al. [4] have found that placental mitochondria were the main source of oxidative stress, as well as the targets of oxidative stress. At present, research about AGEs and oxidative stress are more concentrated in atherosclerosis and diabetes, and has not been directed at placental trophoblast mitochondria oxidative stress. Therefore, this study was designed to study the influence of AGEs on placental trophoblast mitochondria oxidative stress, and to explore the possible pathogenesis which may participate in pre-eclampsia. 


\section{MATERIAL AND METHODS \\ Material \\ Villi tissues}

All the pregnant women were admitted to Fujian Maternity and Children Health Hospital from October 2011 to September 2012 for an abortion. All the subjects had voluntary termination of their pregnancy at 6-8 weeks of gestation. All the subjects were healthy Chinese, 20 to 32 years old, and had normal menstrual periods. Type-B ultrasonic tests confirmed that all the women had intrauterine pregnancies. All the pregnant women were excluded from having pregnancy complications and surgical complications. None of these women had had a spontaneous abortion or missed abortion. We harvested sterile villi by vacuum aspiration for trophoblast cell culture. Informed consent was obtained from each woman and the protocol for this study was approved by the local Institutional Review Board.

\section{Blood samples}

Blood samples were taken from a peripheral vein $(3 \mathrm{~mL}$ of blood) of the arm in the morning before treatment and after overnight fasting. The serum was obtained by centrifugation at 3,000 rpm for $15 \mathrm{~min}$ and stored at $-70^{\circ} \mathrm{C}$ until assayed. Pre-eclampsia was diagnosed by a clinician and classified according to the relevant literature [5]. The clinical features of the subjects are shown in Table 1. Patients with pregnancy complications and surgical complications were excluded from the study. All subjects were single pregnancies.

\section{The preparation of AGEs-human serum albumin}

We prepared the AGEs-human serum albumin according to the literature [6]. There were no toxins in the samples as determined by fluorescence spectrophotometer analysis and enzyme-linked immunosorbent assay (ELISA). Samples were filter sterilized using $0.22 \mu \mathrm{m}$ pore filters.

\section{Methods \\ Primary culture of trophoblast cells and identification \\ We cultured human first trimester trophoblast cells by digestion as previously described [7]. We completely cleared the decidua from the villi tissues, and then repeat- edly washed with PBS to remove the blood clots and impuri- ties. Tissues were cut at the intermediate shaft section and we removed the intermediate shaft section, then cut the}

villi tissues into $1 \mathrm{~mm}^{3}$ pieces and then collected them into centrifuge tubes. Enzymes were added to the centrifuge tubes ( $1: 1$ volume mixed with $0.25 \%$ trypsin and $0.1 \%$ type I collagenase), and the tissues digested in a $37^{\circ} \mathrm{C}$ water bath for $25 \mathrm{~min}$. After that, we added Dulbecco's Modified Eagle Medium: Nutrient Mixture F-12 (DMEM/F12) containing 10\% fetal bovine serum (FBS) to terminate digestion. The enzyme digestion was then filtered with a 200 mesh stainless steel screen, we discarded the supernatant and collected the digested tissue, after centrifugation at 3,000 rpm for $15 \mathrm{~min}$. We inoculated cells into culture at $37^{\circ} \mathrm{C}$ in a $5 \% \mathrm{CO}_{2}$ incubator after suspending them with DMEM/F12 which containing 10\% FBS. Two hours later, we inoculated the remaining cell again with DMEM/F12 which containing 10\% FBS after removing fibroblasts by differential adherent culture.

Trophoblast cells were identified using immunocytochemistry staining. After staining cells with diaminobenzidine (DAB), most of trophoblast cells stained keratin positive and vimentin negative. Trophoblast cells were identified by the Strep Avidin-Biotin Complex (SABC) method using kits made by the American RD Company supplied by the BiYunTian company of JiangSu province. Assays were carried out according to the manufacturer's instructions. Trophoblast cells were fixed with $4 \%$ paraformaldehyde, then $0.5 \%$ Triton was added to rupture the cells followed by $5 \%$ Bovine Serum Albumin (BSA) to stop the reaction. Trophoblast cells were incubated after labeling with dilutions of the first antibodies (anti-cytokeratin 7 and anti-vimentin), then we continued to incubate trophoblast cells after adding the second antibody (goat anti-mouse lgG) without washing. The trophoblast cells were then stained with DAB and counterstained with hematoxylin before step by step alcohol dehydration. Xylene was used to make the trophoblast cells transparent and neutral gum to mount the coverslips. Slides were observed and photographed under a light microscope.

\section{Cell groups}

Pre-eclampsia group 1: trophoblast cells cultured with medium which contains $10 \%$ pre-eclampsia autologous maternal serum for 24 hours then assayed for the levels of mitochondrial oxidative stress damage. The trophoblast cells were divided into two groups; a pre-eclampsia group 2 and an AGEs group. The pre-eclampsia group had cells cultured with pre-eclampsia autologous maternal serum, and cells

\begin{tabular}{|l|c|c|c|c|c|c|c|c|}
\hline \multicolumn{3}{|l|}{ Table 1. The clinically relevant indicators of pregnant women } \\
\hline Group & Case & $\begin{array}{c}\text { Age } \\
\text { (years) }\end{array}$ & $\begin{array}{c}\text { Gestational age } \\
\text { (weeks) }\end{array}$ & $\begin{array}{c}\text { Prepregnancy BMI } \\
{\left[\mathbf{k g} / \mathbf{m}^{2}\right]}\end{array}$ & $\begin{array}{c}\text { Pregnancy BMI } \\
{\left[\mathbf{k g} / \mathbf{m}^{2}\right]}\end{array}$ & \multicolumn{3}{|c|}{ Blood pressure } \\
\hline $\begin{array}{l}\text { Severe } \\
\text { pre-eclampsia }\end{array}$ & 30 & $27.4 \pm 3.3$ & $37.2 \pm 2.0$ & $19.9 \pm 1.2$ & $25.5 \pm 1.2$ & $159.4 \pm 5.5$ & $97.6 \pm 3.2$ & $118.2 \pm 2.5$ \\
\hline
\end{tabular}


in the AGEs group were incubated with $600 \mathrm{mg} / \mathrm{L}$ AGEs for 16 hours before assaying the levels of mitochondrial oxidative stress damage. The two groups were cultured for the same time.

\section{Detection of mitochondrial oxidative stress damage}

Detection the mitochondrial membrane potential

We detected the mitochondrial membrane potential by the Mitochondrial membrane potential assay kit (JC-1) (American AAT Bioquest Inc. Company) supplied by the BiYunTian company of JiangSu province. We incubated $5 \times 10^{4} \mathrm{~mL}^{-1}$ viable trophoblasts in 6-well culture plates with DMEM/F12 which containing 10\% FBS. When the cells covered $80 \%$ to $90 \%$ of the plate we added different serums for 24 hours according to the experimental groups. We added $20 \mu$ l of Carbonylcyan idemchlorophenylhydrazone (CCCP) into a non-drug well as the positive control. The plate was washed with PBS 20 minutes later. Then we added $1 \mathrm{~mL}$ of DMEM/F12 which containing $10 \% \mathrm{FBS}$ and $1 \mathrm{~mL}$ of JC-1 staining working solution to each well and incubated the cells at $37^{\circ} \mathrm{C}$, in a $5 \% \mathrm{CO}_{2}$ incubator for 20 minutes. After washing the plate with $4^{\circ} \mathrm{C} 1 \times \mathrm{JC}-1$ buffer twice, we added $2 \mathrm{~mL}$ of DMEM/F12 which containing $10 \%$ FBS. We photographed trophoblast cells under a fluorescence microscope to observe and calculate the relative proportion of red and green fluorescence.

Measure the activity of mitochondrial permeability transition pore

Six-well plates were seeded with $5 \times 10^{4} \mathrm{~mL}^{-1}$ viable trophoblast cells. When the cells covered $80 \%$ to $90 \%$ of the plate different serums were added according to the experimental groups and the cells cultured for 24 hours. Experimental procedures followed the instructions in the mitochondrial permeability transition pore (MPTP) fluorescence detection kits (American Genmed Scientifics Inc. Company) supplied by the JieMei Company Ltd. The cells were observed under a fluorescence microscope and fluorescence microplate reader RFU values were assigned. Cells were tested once every $5 \mathrm{~min}$ for $30 \mathrm{mins}$ to calculate the MPTP pore activity.

\section{Measuring the expression of mitochondrial DNA}

We extracted genomic DNA using a genomic DNA extraction kit from the RD Company supplied by the JieMei Company. The extraction and assays were carried out according to the kit instructions. The primers and probes were designed by Express software version 2.0 (Applied Biosystems, Foster City, CA). The sequences were derived from the literature [8]. The sequence of mitochondrial DNA primers was referenced using the Human Mitochondrial DNA Revised Cambridge Reference Sequence. The primer gene is mtDNA whose sequences fragment length is $264 \mathrm{bp}$. The sequence of upstream primer is 5'-ACG ACC TCG ATG TTG AAT C-3'. The sequence of downstream primer is $5^{\prime}-\mathrm{GCT}$ CTG CCA TCT TAA CAA ACC-3'. The sequence of the probe is 5'-FAM-TTC AGA CCG GAG TAA TCC AGG TCG-TAMRA-3'. The reference gene is the $28 \mathrm{~S}$ rRNA whose sequences fragment length is $102 \mathrm{bp}$. The sequence of the upstream primer is 5'-TTA AGG TAG CCA AAT GCC TCG-3'. The sequence of downstream primer is 5'-CCT TGG CTG TGG TTT CGC T-3'. The sequence of the probe is 5'-FAM-TGA ACG AGA TTC CCA CCT GTC CCT-ACC TAC TAT C-TAMRA-3'. The conditions used for $P C R$ amplification were a 2 min reaction at $50^{\circ} \mathrm{C}$ to denature, a $10 \mathrm{~min}$ reaction at $95^{\circ} \mathrm{C}$ to activate the AmpliTaq Gold DNA polymerase, and 40 cycles of $10 \mathrm{sec}$ at $95^{\circ} \mathrm{C}$ and $40 \mathrm{sec}$ at $60^{\circ} \mathrm{C}$. To standardize the mitochondrial DNA content, a comparative analysis was performed by amplifying an endogenous control, $28 \mathrm{~S}$ rRNA. The amount of target relative to endogenous reference was calculated as $2^{-\Delta \Delta c t}$.

\section{Statistical analysis}

Data are presented as mean \pm SD. Comparisons between two groups were performed using an unpaired t-test. Comparisons among groups were performed using one-way ANOVA with the Levene test, followed by the multiple comparisons among four groups using the Dunnett T3 method. Pearson correlation analysis was adopted to assess the correlations between serum AGEs and FFA. A $P$-value $<0.05$ was regarded as statistically significant. All calculations were carried out with the Statistical Package for Social Science Software (SPSS17.0).

\section{RESULTS}

\section{The general situation of the pregnant women}

The maternal age, gestational age, pre-pregnancy body mass index (BMI), pregnancy BMI, systolic pressure (SP), diastolic (DP) and mean arterial pressure (MAP) of severe pre-eclampsia women are shown in Table 1.

\section{Primary culture of trophoblast cells and identification}

Trophoblast cells were directly observed under an inverted microscope. They had epithelioid cell morphology, mostly irregular polygonal in shape, abundant cytoplasm, and large ovoid nuclear, and grew as a monolayer (Fig. 1A). Immunocytochemistry showed that the cytoplasm of trophoblast cells were keratin positive (Fig. 1C) and vimentin negative (Fig. 1C), cell purity of $95 \%$.

\section{Results of each group's mitochondrial membrane potential}

The results of each group's mitochondrial membrane potential are shown in Figure 2. The mitochondrial membrane po- 

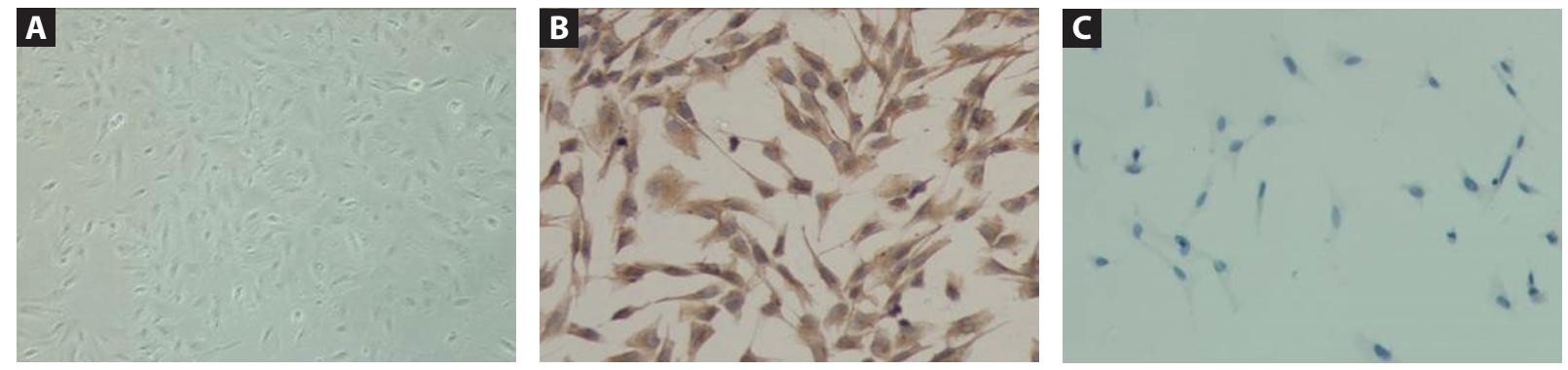

Figure 1. Primary culture trophoblast cells and identification
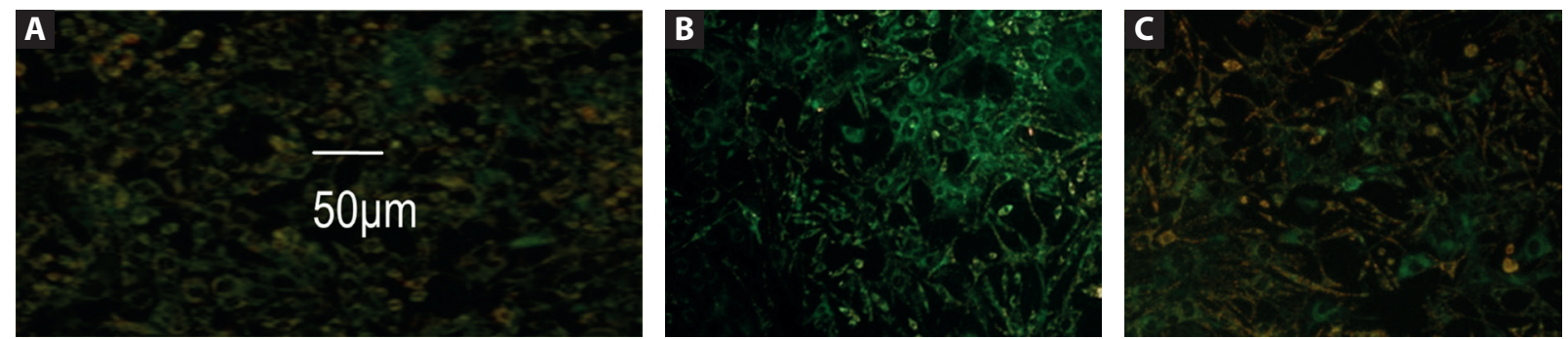

Figure 2. Fluorescence microscopy detected the mitochondrial membrane potential (Bar $=50 \mu m$, magnification $\times 100)$. A. Pre-eclampsia group 1 ; B. AGEs group; C. Pre-eclampsia group 2

tential of the AGEs group was significantly decreased; the membrane potential was significantly depolarized. The vast majority of trophoblast cells showed green fluorescence. The mean portion of mitochondrial membrane potential depolarization in the pre-eclampsia group 1 is $64.27 \pm 2.27 \%$, and the in the AGEs group it was $94.70 \pm 0.95 \%$. In the pre-eclampsia group 2 it was $66.17 \pm 1.94 \%$. The mean mitochondrial membrane potential depolarization in the AGEs group was significantly higher than both pre-eclampsia group 1 and group $2(P<0.05)$. However, no significant difference was found between the pre-eclampsia group 1 and pre-eclampsia group 2 .

\section{Results of activity of mitochondrial permeability transition pore of each group}

The results of the activity of the mitochondrial permeability transition pores of each group are as follows
(Figs. 3 and 4). The AGEs group shows that green fluorescence enhancement for calcein continued into the mitochondrial aggregation and the quenching rate was very low, indicating an increased opening of the mitochondrial membrane channel pores. The green fluorescence intensity in the AGEs group was higher than in pre-eclampsia group 1 and group 2. This showed that the opening of the mitochondrial membrane channel pore in the AGEs group was greater than in either pre-eclampsia group 1 or group 2.

\section{Results of the expression of mtDNA}

The results of the expression of mtDNA for each group are shown in Table 2 . The mean relative mtDNA expression in the AGEs group, $2.49 \pm 0.15$, was significantly higher than in the pre-eclampsia group 1, $2.10 \pm 0.03$, and pre-eclampsia
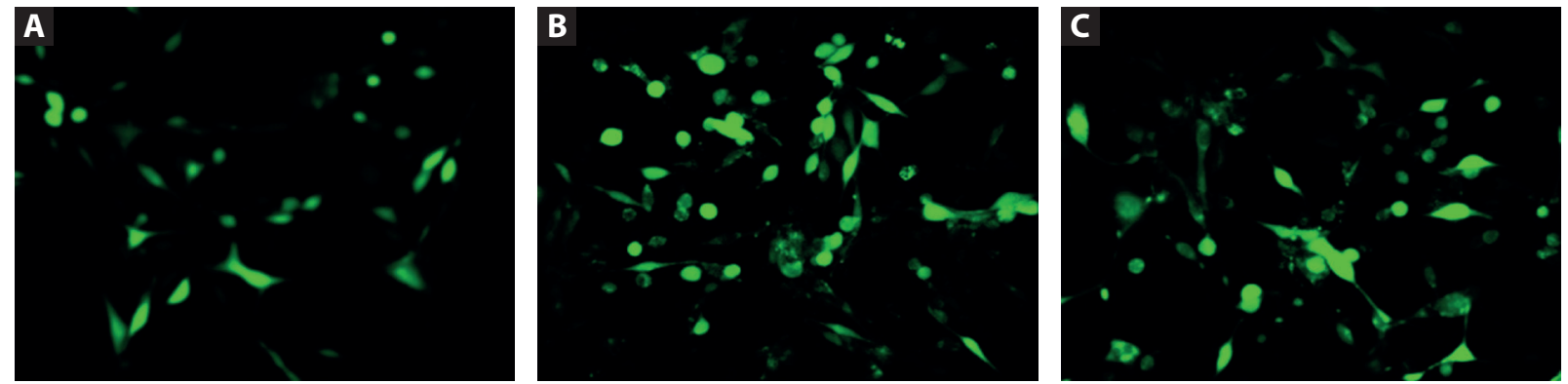

Figure 3. Fluorescence microscopy detected the activity of the mitochondrial permeability transition pore, $(B a r=50 \mu m$, magnification $\times 100)$, A. Pre-eclampsia group 1; B. AGEs group; C. Pre-eclampsia group 2 
Table 2. Comparison of CT values in each group and the relative content of mtDNA

\begin{tabular}{|l|c|c|c|}
\hline Groups & 28S rRNAC & mtDNAC $_{\mathbf{T}}$ & mtDNA/28S rRNA \\
\hline AGEs & $15.64 \pm 0.07$ & $16.96 \pm 0.09$ & $2.49 \pm 0.15^{*}$ \\
\hline Pre-eclampsia group 1 & $15.79 \pm 0.09$ & $16.86 \pm 0.08$ & $2.10 \pm 0.03$ \\
\hline Pre-eclampsia group 2 & $15.83 \pm 0.10$ & $16.92 \pm 0.03$ & $2.14 \pm 0.11$ \\
\hline
\end{tabular}

*P $<0.05$

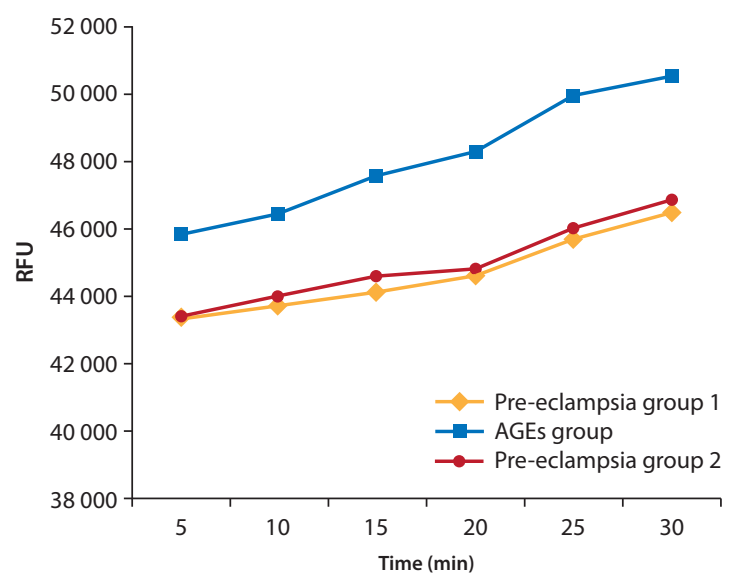

Figure 4. Fluorescence microplate reader detected the activity of the mitochondrial permeability transition pores

group 2, 2.14 \pm 0.11 . However, no significant difference was found between the pre-eclampsia group 1 and pre-eclampsia group 2 (Fig. 5).

\section{DISCUSSION}

\section{Biological activity of AGEs}

AGEs accumulate slowly during natural aging. However, AGEs are dramatically increased in some disorders; especially in those patients who suffer from oxidative stress with conditions such as diabetes, rheumatoid arthritis, systemic lupus erythematosus, Alzheimer's disease, or in cigarette smokers. Olier et al. [9] have found that the expression of AGEs in maternal serum of patients with pre-eclampsia was significantly higher when compared to the expression in normal pregnant women and non-pregnant women. Gueds et al. [10] have also shown that the accumulation of AGEs can cause oxidative stress in the placenta and placental vascular dysfunction, and thus lead to pre-eclampsia. Chekir et al. [1] found that expression of AGEs in maternal serum and in the placenta of patients with pre-eclampsia was significantly higher when compared to the expression during normal pregnancy, and markers of oxidative stress damage in the placenta were significantly increased. Such results could suggest that high expression of AGEs may have as important a role in the pathophysiology of pre-eclampsia as oxidative stress; although the exact mechanism is still unknown.

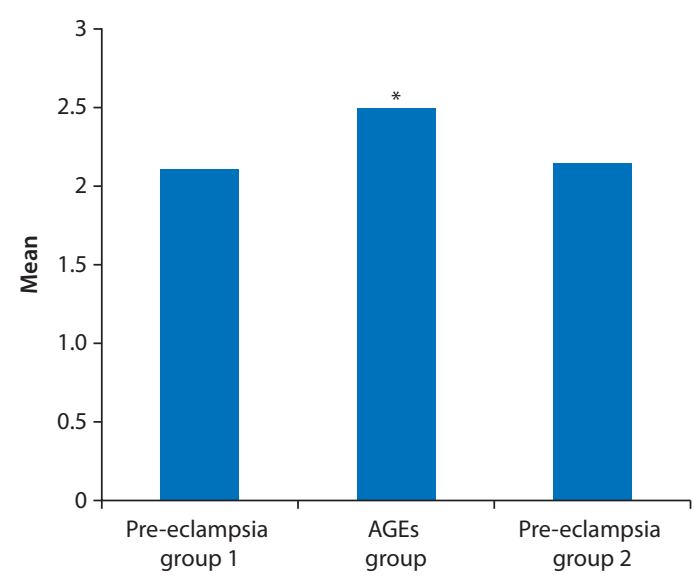

Figure 5. Comparison of the relative mtDNA expression of each group AGEs group compared with the other two groups; ${ }^{*} \mathrm{P}<0.05$

\section{Mitochondrial oxidative stress and pre-eclampsia}

Oxidative stress (OS) is an imbalance between pro-oxidant production and antioxidant defenses that produces a large number of reactive oxygen species (ROS). In normal pregnancy, oxidative production and antioxidant defenses remain relatively balanced and prevent oxidative stress. Placental perfusion decreases in the late trimester, while the body's compensatory mechanisms reperfusion the placenta, producing ROS resulting in oxidative stress. Mitochondria are the source of energy production, but also the main site of endogenous ROS production. Zusterzeel et al. [4] have found that placental mitochondria were the main source of oxidative stress, as well as the targets of oxidative stress. ROS are extremely active. Too many ROS can cause mitochondrial oxidative stress damage, which would change the structure and function of trophoblast cells, leading to placental ischemia and hypoxia that could participate in the pathogenesis of pre-eclampsia. There are many MPTP on the mitochondria membrane. The periodic opening of MPTPs plays an important role keeping an electrochemical balance within mitochondria. Loor et al. [11] found that ROS could induce MPTP opening by the oxidation of sensitive points on the MPTPs. Once the opening of MPTPs is increased, the mitochondrial permeability would also be increased. That would result in mitochondrial depolarization and a lowering or disappearance of the mitochondrial membrane potential. Yet a lower mitochondrial membrane 
potential would also promote opening the MPTPs, which then forms a vicious cycle [12]. Excess ROS can fracture the bond which crosslink DNA with protein, and thus would damage DNA. The mtDNA has fewer protected areas than nuclear DNA. So mtDNA is more susceptible to ROS damage. Yan et al. [13] have found that women with pre-eclampsia had higher levels of oxidative stress damage in placental mitochondria than did controls. This included a lower mitochondrial membrane potential, increased mitochondrial permeability, and higher levels of mtDNA.

\section{AGEs and mitochondrial oxidative stress}

Many studies have found that AGEs can stimulate ROS production that induces mitochondrial oxidative stress damage. Yu et al. [14] have found that in in vitro studies AGEs can stimulate the human umbilical vein endothelial cells to generated ROS, and the amount of ROS increases with the AGE concentration and incubation time. Maximal ROS were generated when endothelial cells were incubated with 600 mg/L AGEs for 16 hours. Any further increase of AGEs concentration or incubation time had no significant change in ROS volume. Aline et al. [2] have also found that AGEs can stimulate ROS production, inducing mitochondrial oxidative stress damage to the mitochondria structure that inhibits electron transfer. This resulted in lower mitochondrial membrane potential and damage to the mitochondrial energy metabolism. Tetsuro et al. [3] have also shown that exogenous AGEs can act on mast cells, resulting in a mitochondrial $\mathrm{Ca}^{2+}$ concentration overload, mitochondrial membrane potential collapse and mitochondrial structural integrity damaged. Mitochondrial permeability increased, cytochrome $C$ was released and caspases-3/7 were activated which induced mast cell apoptosis. Huang et al. [15] have discovered that AGEs increases intracellular ROS generation, increases oxidative stress damage that leads to pre-eclampsia, although the exact mechanism remains unclear.

This present study showed that in the AGEs group the mitochondrial membrane potential was significantly lower, the MPTPs were significantly more open, and the expression of mtDNA was significantly increased. The mitochondrial oxidative stress damage in the AGEs groups was significantly increased when compared to both pre-eclampsia group- 1 and group-2. This study also shows that mitochondrial oxidative stress damage in the AGEs group was significantly greater when compared to the group without AGEs. The expression of AGEs in maternal serum and in the placenta of the patients with pre-eclampsia was significantly higher when compared to the expression during normal pregnancy [1]. These observations suggest that AGEs maybe participate in the pathophysiology of pre-eclampsia by increasing oxidative stress damage.

\section{CONCLUSIONS}

It was found in this study that AGEs could increase the levels of oxidative stress damage. This included lower mitochondrial membrane potential, increased mitochondrial permeability, and higher levels of mtDNA. This implies that AGEs may participate in the pathophysiology of pre-eclampsia by increasing oxidative stress damage. This will provide a new perspective for the pathogenesis and prevention of pre-eclampsia.

\section{Acknowledgments}

This work was supported by the Natural Science Foundation of Fujian Province of China. The Fund No. is 2012J01313.

\section{Disclosure}

The authors declare no potential conflict of interest with respect to the authorship and publication of this article.

\section{REFERENCES}

1. Chekir C, Nakatsuka M, Noguchi S, et al. Accumulation of advanced glycation end products in women with preeclampsia: possible involvement of placental oxidative and nitrative stress. Placenta. 2006; 27(2-3): 225-233, doi: 10.1016/j.placenta.2005.02.016, indexed in Pubmed: 16338468.

2. Remor AP, de Matos FJ, Ghisoni K, et al. Differential effects of insulin on peripheral diabetes-related changes in mitochondrial bioenergetics: involvement of advanced glycosylated end products. Biochim Biophys Acta. 2011; 1812(11): 1460-1471, doi: 10.1016/j.bbadis.2011.06.017, indexed in Pubmed: 21767639.

3. Yoshimaru T, Suzuki Y, Inoue T, et al. Extracellular superoxide released from mitochondria mediates mast cell death by advanced glycation end products. Biochim Biophys Acta. 2008; 1783(12): 2332-2343, doi: 10.1016/j.bbamcr.2008.08.010, indexed in Pubmed: 18822320

4. Zusterzeel PL, Rütten $\mathrm{H}$, Roelofs HM, et al. Protein carbonyls in decidua and placenta of pre-eclamptic women as markers for oxidative stress. Placenta. 2001; 22(2-3): 213-219, doi: 10.1053/plac.2000.0606, indexed in Pubmed: 11170826.

5. Leveno KJ, Cunningham FG, Gant NF. Williams manual of obstetrics, 21st edition. McGraw-Hill 2003

6. Bierhaus A, Illmer T, Kasper M, et al. Advanced glycation end product (AGE)-mediated induction of tissue factor in cultured endothelial cells is dependent on RAGE. Circulation. 1997; 96(7): 2262-2271, indexed in Pubmed: 9337199.

7. Liu HP, Wang RG, Li CM. Establishment of in vitro culture system of human archae-generation trophoblasts. Practical Preventive Medicine. 2006; 13(5): 1109-1111.

8. Cha KY, Lee $\mathrm{SH}$, Chung HM, et al. Quantification of mitochondrial DNA using real-time polymerase chain reaction in patients with premature ovarian failure. Fertil Steril. 2005; 84(6): 1712-1718, doi: 10.1016/j. fertnstert.2005.06.036, indexed in Pubmed: 16359970.

9. Oliver EA, Buhimschi CS, Dulay AT, et al. Activation of the receptor for ad vanced glycation end products system in women with severe preeclampsia. J Clin Endocrinol Metab. 2011; 96(3): 689-698, doi: 10.1210/jc.20101418, indexed in Pubmed: 21325454.

10. Guedes-Martins L, Matos L, Soares A, et al. AGEs, contributors to placental bed vascular changes leading to preeclampsia. Free Radic Res. 2013; 47 Suppl 1: 70-80, doi: 10.3109/10715762.2013.815347, indexed in Pubmed: 23796030

11. Loor $\mathrm{G}$, Kondapalli J, Iwase $\mathrm{H}$, et al. Mitochondrial oxidant stress triggers cell death in simulated ischemia-reperfusion. Biochim Biophys Acta. 2011; 1813(7): 1382-1394, doi: 10.1016/j.bbamcr.2010.12.008, indexed in Pubmed: 21185334.

12. Fang $X Y, L i$ YG, Lin NY. Mitochondrial permeability transition pore and its mechanism of influence by the mitochondrial ATP-sensitive potassium channel opening. Journal of Shantou University Medical College. 2012; 25(1): 61-64. 
13. Yan Jy, Xu X. [Relationships between concentrations of free fatty acid in serum and oxidative-damage levels in placental mitochondria and preeclampsia]. Zhonghua Fu Chan Ke Za Zhi. 2012; 47(6): 412-417, indexed in Pubmed: 22932105.

14. Yu SY, Huang L, Song MB. Increased reactive oxygen species in endothelial cells stimulated by advanced glycation end products mediated by NADPH oxidase. Chinese Journal of Arteriosclerosis. 2008; 16(11): 857-860.

15. Huang $\mathrm{OT}$, Zhang $\mathrm{M}$, Zhong $\mathrm{M}$, et al. Advanced glycation end products as an upstream molecule triggers ROS-induced sFlt-1 production in extravillous trophoblasts: a novel bridge between oxidative stress and preeclampsia. Placenta. 2013; 34(12): 1177-1182, doi: 10.1016/j. placenta.2013.09.017, indexed in Pubmed: 24144948. 